\title{
Data fusion used in multispectral system for critical protection
}

\author{
R. Dulski, M. Kastek, G. Bieszczad, \\ P. Trzaskawka \& W. Ciurapiński \\ Institute of Optoelectronics, Military University of Technology, \\ Warsaw, Poland
}

\begin{abstract}
The paper presents a multispectral protection system for critical infrastructure. The system consists of a vision camera, an IR camera and radar. The radar data are utilized to establish the location of a potential intruders and to control an observation area for system cameras. A final decision from system is evaluated using image data. The method of data fusion used has been introduced. It consists of image fusion algorithm which allows visualizing and tracking intruders under any conditions. The multisensor construction of the protection system allows one to achieve significant improvement of detection probability of intruder and reduce false alarms.

Keywords: multisensor security system, radar, VIS camera, IR camera, data fusion.
\end{abstract}

\section{Introduction}

Recent events like terrorist attacks on 11.09.2001 and later-on conflicts in Iraq and Afghanistan, the necessity to protect military bases, convoys and patrols gave serious impact to the development of security systems for protection of strategic installations. The changes are visible also in the views and protection methods, taking into account the increased skill level of terrorist groups and the availability of high-tech sensors. Popular and widely-used concepts of perimeter protection with double fence and zone sensors [1-3] supported by area illumination and VIS cameras is now phased out in favor of multi-sensor platforms, integrating (i) day/night cameras for contrast detection, (ii) uncooled thermal cameras for thermal contrast detection and (iii) millimeter-wave radars 
detecting the electromagnetic radiation reflected from target. Effective ranges of detection, recognition and identification for a human or a vehicle-type target depend on the class of the sensor used and the observed scene itself [4-6]. The key element of a multi-sensor security system is adaptive, intelligent image analysis. The use of image fusion methods make it possible to automatically detect the intruder and launch automatic tracking of its movement. The gigabit Ethernet is used as the core telecommunication network of the system, which enables building both large, wide-area systems and small, single unit security installations as well. The important advantage of multi-sensor system is scalability, which applies to both observation range and the size of protected area.

\section{Concept of security system}

The sensor platform integrating millimeter-wave radar (STS-1400 by ICX) and multi-sensor camera system (PTZ 50MS by FLIR Systems) was chosen as a main element of the proposed solution. The area coverage of such platform is schematically presented in Fig.1.

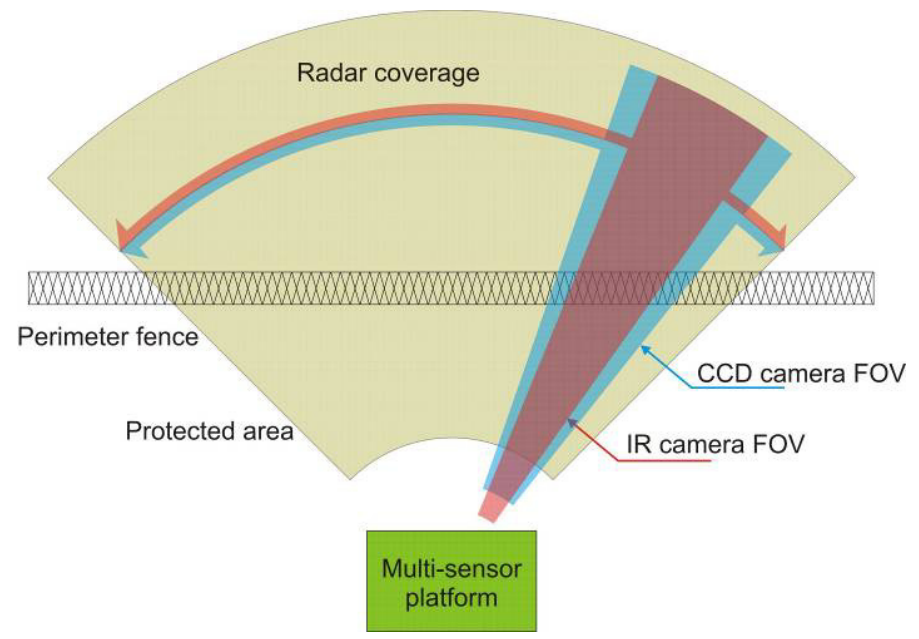

Figure 1: Observation areas of particular components of a multi-sensor platform.

Radar sensor has the longest detection range of 800 meters, which makes it the primary sensor of the security system. During the operation of the system, the radar data are analyzed and the intruder location is send to actuators driving the cameras. In automatic mode the cameras track the intruder. The recorded images are sent to the image analysis unit, which uses the image fusion method. The separate unit combines the data from all the sensors and presents them on the operator's console. 


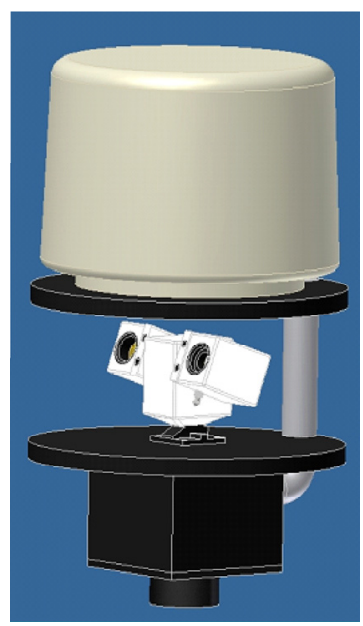

Figure 2: General view of the multi-sensor platform: radar dome (above) and cameras (below).

The sensor platform presented in Fig 2. is mounted at the height that ensures the required detection ranges.

\section{System components}

Radar STS 1400 can detect a human being moving at the velocity of about $2 \mathrm{~m} / \mathrm{s}$ at the range of $800 \mathrm{~m}$, under normal atmospheric conditions. The movement capabilities of the cameras (visible and infrared) are $+30 \%-45^{\circ}$ in elevation and $360^{\circ}$ in azimuth. Sony's FCB EX-980SP visual camera used in the system has a CCD imager boasting 800000 effective pixels, zoom lens with a FOV changing from $42^{\circ}$ to $1.6^{\circ}$ and $26 \mathrm{x}$ electronic zoom. These characteristics mean that a human can be detected from $880 \mathrm{~m}$, and a vehicle from about $2.2 \mathrm{~km}$. Second camera is a thermal camera unit with uncooled focal plane array (FPA). It has 8 - $14 \mu \mathrm{m}$ working range, $320 \times 256$ pixels resolution, FOV $14^{\circ}$ (horizontal) $\mathrm{x}$ $10^{\circ}$ (vertical) and $2 \mathrm{x}$ electronic zoom. It can detect a human from 650 meters and a vehicle from about 2 kilometers.

During preliminary tests, aimed at evaluation of effectiveness of the data fusion algorithms, the platform was mounted $6 \mathrm{~m}$ above the ground level. As a result the effective ranges for human detection of the particular components were: radar - 780 meters, visual camera - 760 meters and IR camera - 480 meters. The ranges were estimated on the basis of technical specifications of these devices. The software instrumentation of the system is based on FLIR Nexus system [7], which has software interface between cameras and ICX radar. However, the Nexus system does not fulfil all assumed functions. Image data fusion, target detection and tracking and sensor data merging are performed by separate software. The software analyzes the images and independently detects intruders and the results are displayed with confirmation of the alarm. The data 
fusion system performs synthesis of numerical data and displays information on the numerical map on the operator's interface. The system has the possibility of automatic testing and signalization of possible mechanical, software, communication or power supply failures.

\section{Data fusion concept}

The brief description of the operation of data fusion algorithm is presented below. At the moment of possible intruder detection by the radar system, the range an direction data are transferred to the camera positioning system, adjusting its line of sight accordingly. The field of view of both cameras are matched to cover similar areas, as shown in Fig.3.
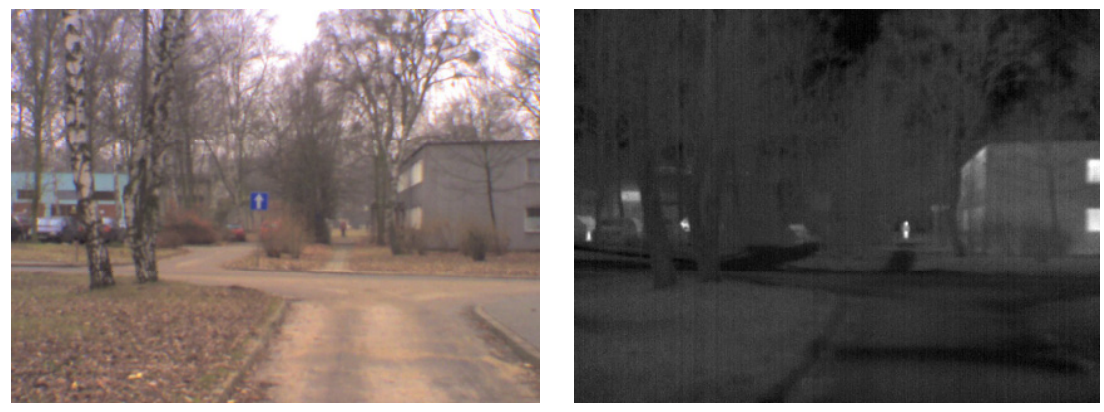

Figure 3: $\quad$ Images of observed scene from VIS and IR cameras.
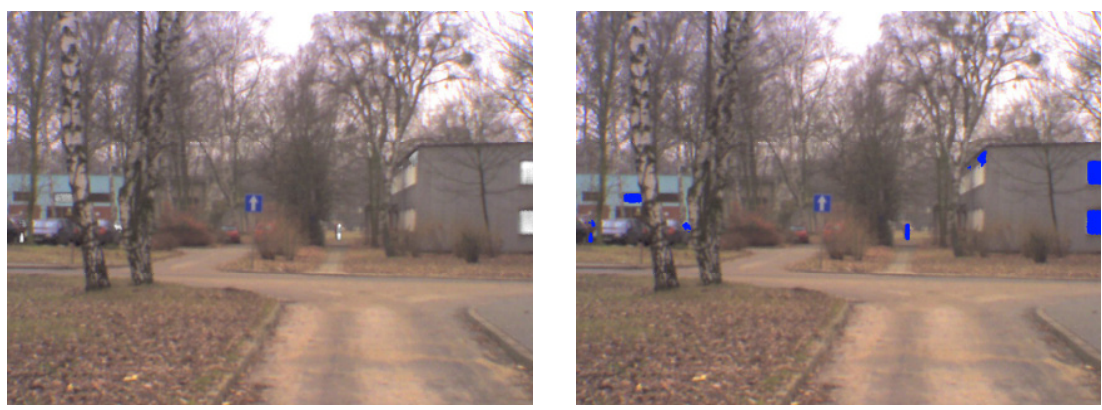

Figure 4: $\quad$ Targets selected after the application of mask.

Each image is then analyzed, and "suspected" areas are marked out. Thermal image is then binarized with pre-defined threshold and as a result the selection mask is obtained. The mask is created in such a way, that the area of interest is brought to a high level and appears as white whereas all the remaining scene is brought down to a "low" level and consequently is black. In order to distinguish objects from background, the adaptive temperature threshold is calculated and everything below that threshold is treated as background. The algorithms used to determine the threshold brightness level are, for visual images, usually based on 
image histogram evaluation. In case of thermal images, probability density function is more appropriate, which shows the probability of occurrence of given temperature value in the thermal image. The function was discretized in temperature domain and as a result the histogram was obtained, showing the probability of temperature ranges to appear in the image. However, during initial evaluations the simple approach of fixed threshold was applied, which was arbitrary assumed at $60 \%$ of image dynamic range.

In the next step of image processing the mask is overlaid onto visual image and the potential targets are marked with appropriate colors (Fig.4).

The resulting image is created by merging the visual image transformed according to above procedure with IR image. The final stage of image processing is target tracking.

Multi-sensor security system is expected to have fast guaranteed reaction time, therefore it can be treated as a real-time system. Consequently, emphasis was put on the suitable tracking method capable of real-time operation. Several tracking algorithms were considered, like target feature-tracking Mean-Shift algorithm [8] and Sum-of-Squared-Differences (SSD) gradient algorithm [9, 10]. During the simulations, Mean-Shift approach was inefficient when the tracked object covered small number of pixels. As a result, modified version of SSD algorithm was adopted to perform the tracking task in a security system.

Gradient SSD algorithm analyzes the differences between two consecutive frames to find the target location. The target movement is estimated by calculating spatial and temporal gradients.

SSD coefficient defines the difference between two consecutive fragments of the image. Both analyzed fragments have to be of the same size and they are usually rectangular. Assuming, that the two fragments (called "windows") are $(2 h+1)$ by $(2 h+1)$ in size and that they centers have the coordinates $(x, y)$ and $(u, v)$ respectively, the SSD coefficient can be calculated according to the following relation:

$$
S S D=\sum_{i, j}\left[\left(f_{n-1}(x+i, y+j)-f_{n}(u+i, v+j)\right)^{2}\right],
$$

where:

$i, j \in[-h, h]$ - point coordinates with respect to the centers of compared fragments, $h$ - target size coefficient.

If we assume that the tracked object is present on the $f_{n-1}$ frame and is centered around $(x, y)$ coordinates, then finding this object on the consecutive frame means, that the point $(u, v)$ is to be found, for which the SSD coefficient has minimal value. The point $(u, v)$ is then the center of the tracked object on the $f_{n}$ frame. The search for the minimal value of SSD coefficient is performed in the neighborhood of the past location of tracked object. The size of the search area depends on the assumed object's dynamics. It means, that the frame-to-frame movement range should be estimated. Maximal value of the movement (in pixels) should correspond to the tracking range. The bigger is the tracking area, the more calculations must be performed. To minimize the required number of calculations the frame-to-frame movement should be small. For constant 
observation parameters and object's dynamics it can be accomplished by increasing the frame rate.

As a result of calculations, the values of SSD parameters are obtained for the elements of the image. This set can be presented as a matrix having the same size as the search area. Sample results for a tracked object are presented in Fig.5. The 3-D plot shows the SSD values as the function of the position of corresponding image fragment. Minimal value of SSD coefficient indicates the greatest resemblance to the model object.
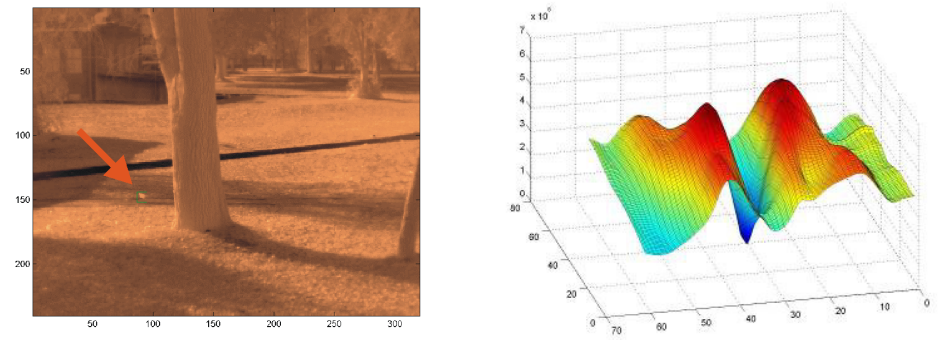

Figure 5: SSD coefficients (right) for the image areas in the neighborhood of tracked object (left).

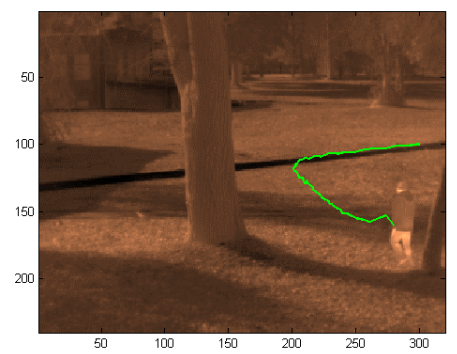

Figure 6: First frame of the tracking sequence and object's trajectory derived from the consecutive frames of the sequence.

The tracking procedure described above is then repeated for the consecutive frames. The area, where the object was found is treated as a reference model object for the movement calculations on the next frame. If the algorithm derives the next location incorrectly, then for the following calculations an empty area (not containing the object) becomes the reference model. The model object is updated unconditionally in this algorithm, so it may happen that the original shape is forgotten and the tracking is lost. Similar effect may occur when the object becomes partially covered, and then due to information loss the next location will not be successfully calculated. It is clear then, that this algorithm has good frame-to-frame efficiency, but long-term effectiveness could be less impressive. Small disturbances, coverage, collisions or noise may lead to the loss 
of tracking. Surprisingly, as the tests revealed, the presented algorithm is quite effective when used on thermal images. The example of object's tracking is presented in Fig.6.

At the final stage the data fusion algorithm includes the radar and GPS data for the synthetic visualization on the map of the monitored area.

\section{Conclusions}

The figure below shows the example images obtained as a result of image fusion approach.
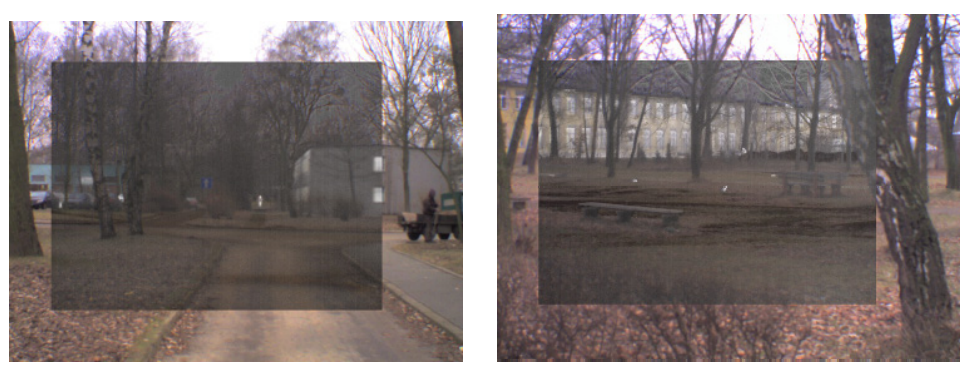

Figure 7: The composite image obtained by image fusion.

By comparing the composite image to the components it was made from, it can be seen that the result is easier to perceive and interpret. The marked targets can be easily pointed out and tracked. The application of multi-sensor platforms in a security system increases its functionality and performance. Radar sensor can detect potential intrusion quicker than a single camera. The fusion of visual and thermal images increases the probability of intruder detection regardless of the time of day or weather conditions [11]. In practical applications the probability of intruder detection is increased and simultaneously the false alarm rate is decreased. The effective tracking algorithm on the basis of thermal images makes it possible to observe intruder's activity and undertake the best possible counteraction.

The developed data fusion algorithm will be further optimized during field tests, taking into account the external conditions and assumed configuration of the security system.

The job is supported by the Polish Ministry of Science and Higher Education in the frame of research program funding plan for the years 2008-2010.

\section{References}

[1] Madura H., Method of signal processing in passive infrared detectors for security systems, WIT Transactions on Modeling and Simulation 46, pp. 757-768, 2007. 
[2] Kastek M., Sosnowski T., Polakowski H., Dąbrowski M., Orżanowski T., Long-range PIR detector used for detection of crawling people, Proceedings of SPIE Vol. 7113, 71131F, 2008.

[3] Kastek M., Sosnowski T, Piątkowski T., Passive infrared detector used for detection of very slowly moving of crawling people, Opto-Electronics Review 16 (3), pp. 328-335, 2008.

[4] Holst C., Testing of infrared imaging systems, JVC, New York 1995.

[5] Dulski, R., Niedziela, T., Verification of the correctness of thermal imaging modelling, Optica Applicata, Vol. XXXI, No 1, 2001, pp. 193-202.

[6] Dulski R., Madura H., Piątkowski T., Sosnowski T., Analysis of a thermal scene using computer simulations, Infrared Physics \& Technology, 49 (2007) pp. 257-260.

[7] NEXUS FLIR Networked Systems, http://www.flir.com/

[8] Isard M., Blake A. "Contour tracking by stochastic propagation of conditional density", in: European Conference on Computer Vision, 1996, pp. 343-356.

[9] Hager G., Belhumeur P., Efficient region tracking with parametric models of geometry and illumination, IEEE Trans. Pattern Anal. Mach. Intell. 20 (10) (1998) pp. 1025-1039.

[10] Fukunaga K., Introduction to Statistical Pattern Recognition. Academic Press, second edition, 1990.

[11] Polakowski H., Dulski R., Firmanty K., Evaluation of sky and clouds IR radiation, Proc. of the Advanced Infrared Technology and Applications AITA 9, Leon, pp. 383 - 386, 2008. 